\title{
Analysis of Temperature Dependence of Dark Current Mechanisms in Mid-Wavelength Infrared pin Type-II Superlattice Photodiodes
}

\author{
Jarosław Wróbel ${ }^{*}$, Elena Plis ${ }^{1}$, Waldemar Gawron, Marcin Motyka ${ }^{2}$, \\ Piotr Martyniuk, Paweł Madejczyk, Andrzej Kowalewski, Mateusz Dyksik², \\ Jan Misiewicz ${ }^{2}$, Sanjay Krishna ${ }^{1}$ and Antoni Rogalski \\ Institute of Applied Physics, Military University of Technology, \\ 2 Kaliskiego St., 00-908 Warsaw, Poland \\ ${ }^{1}$ Department of Electrical and Computer Engineering, Center for High Technology Materials, \\ University of New Mexico, Albuquerque, New Mexico 87106, USA \\ ${ }^{2}$ Institute of Physics, Wroclaw University of Technology, \\ Wybrzeże Wyspiańskiego 27, 50-370 Wrocław, Poland
}

(Received November 21, 2013; accepted February 24, 2014)

Key words: dark current, type II, superlattice, photodiode, differential resistance, infrared detection, high operating temperature, reduced mass, pin structure

We report on the temperature dependence characteristics of mid-wavelength InAs/ $\mathrm{GaSb}$ type-II superlattice photodiodes in a temperature range from 120 to $240 \mathrm{~K}$. The bulk material model with an effective bandgap of superlattice material has been used in the modelling of the experimental data. Temperature and bias-dependent differential resistances have been analyzed in detail owing to the contributing mechanisms that limit the electrical performance of the diodes. The $\mathrm{C} 1-\mathrm{HH} 1$ reduced mass has been estimated from the fitting to the high reverse bias $(<-1.0 \mathrm{~V})$ voltage and given as about $0.015 m_{0}$ in nearly the entire considered temperature range. This value agrees well with much more complex simulations and cyclotron resonance measurements. Obtaining very good results was possible, thanks to the inclusion of series resistance into the calculations. In this paper, we show how to overcome difficulties with the nonlinear problem related to it.

\section{Introduction}

In the middle of the 1970s, investigations on the InAs/GaSb superlattice (SL) near lattice-matched systems were performed as an alternative to the GaAs/AlAs SLs. The ability of the InAs/GaSb material system to achieve small infrared (IR) energy gaps was first realized in 1977.(1) At once, the type II band offset in this system was revealed ${ }^{(2)}$ and the potential use of this system in IR photoelectronics was recognized. At present, ${ }^{\bar{*}}$ Corresponding author: e-mail: jarekwrobel@wat.edu.pl 
the InAs/GaSb strained layer SLs (SLSs) are considered as alternatives to $\mathrm{HgCdTe}$ IR material systems ${ }^{(3)}$ and a candidate for third-generation IR detectors ${ }^{(4)}$ as well as in high operating temperature (HOT).$^{(5)}$

In this paper, we focused on one of the possible constructions of the mid-wavelength IR (MWIR) InAs/GaSb SL photodiodes, namely, pin structures. We concentrate here on a dark current modelling to point out which components limit the device performance at a determined bias voltage and temperature regime. Because counting nonequilibrium states and current flow direct from the SL band structure (even for the ideal crystal approximation) is rather complicated (see e.g. ref. 6), bulklike modelling is often used.(7-11) In most cases, researchers have focused on a cryogenic application and region close to the zero bias voltage, where the series resistance $\left(R_{\text {series }}\right)$ is usually neglected, owing to its small value (compared with $R_{\text {device}}$ ). In this paper, we extend analyses up to the HOT conditions, from 120 to $240 \mathrm{~K}$, and bias voltage from -1.6 to $+0.3 \mathrm{~V}$. We show how to overcome the difficulties related to taking into account $R_{\text {series }}$, in the simplest situation, i.e., with no exclusion effect. Finally, we compare some of the fitting results, i.e., $m^{*}$ red for C1-HH1 transition [see eq. (7)], with available literature data.

\section{Growth and Device Design}

The representative sample presented in this work is the InAs/GaSb SLS pin detector with the SU-8 passivation fabricated at the Center for High Technology Materials, University of New Mexico, Albuquerque, New Mexico. The device structure was grown on Te-doped epiready (100) GaSb substrates. It consists of 100 periods of ten monolayers (10 MLs) of InAs:Si $\left(n=4 \times 10^{18} \mathrm{~cm}^{-3}\right) / 10$ MLs of GaSb as the bottom contact layer. This was followed by 50 periods of graded n-doped 10 MLs of InAs:Si/10 MLs of GaSb, 350 periods of absorber, 25 periods of 10 MLs of InAs:Be $\left(p=1 \times 10^{18} \mathrm{~cm}^{-3}\right) / 10 \mathrm{MLs}$ of $\mathrm{GaSb}$, and finally, 17 periods of $10 \mathrm{MLs}$ of InAs:Be $\left(p=4 \times 10^{18} \mathrm{~cm}^{-3}\right) / 10 \mathrm{MLs}$ of GaSb, which formed a p-type contact layer.

A normal-incidence single-pixel mesa photodiode, with a $450 \times 450 \mu \mathrm{m}^{2}$ electrical area, was fabricated by photolithography and inductively coupled plasma etching. Ohmic contacts were made by depositing Ti/Pt/Au on the contact layers. For details, see ref. 12 .

\section{Experimental Results}

For the experiments, we have chosen two samples with the same design (see Fig. 1 ) and electric areas, but essentially different $R_{\text {shunt }}$ and $R_{\text {series }}$. This approach could give us information that the presented model is independent of these resistance values (see discussion in $\S \S 4$ and 5). The dark current-voltage characteristics were measured in the temperature range of 120-240 K using a Keithley 2400 Source Meter (see Fig. 2). The differential resistances at different temperatures were calculated on the basis of the dark current data and presented in Fig. 3. The explanation of the shapes of these characteristics and the description of the mechanisms that govern current flow are provided by theoretical modelling (see $\S 4$ ). 


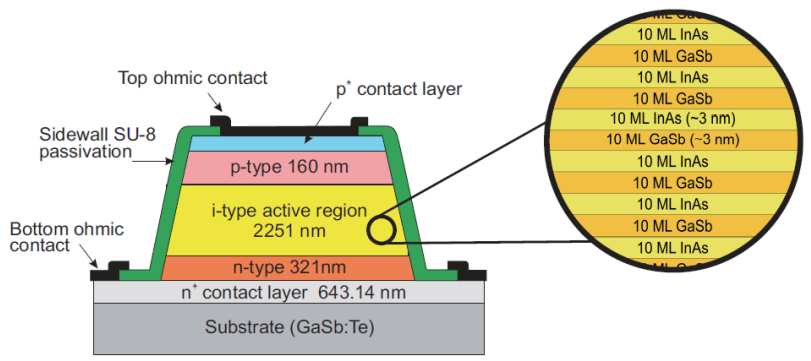

Fig. 1. (Color online) Single-pixel InAs/GaSb superlattice pin photodiode (not to scale).
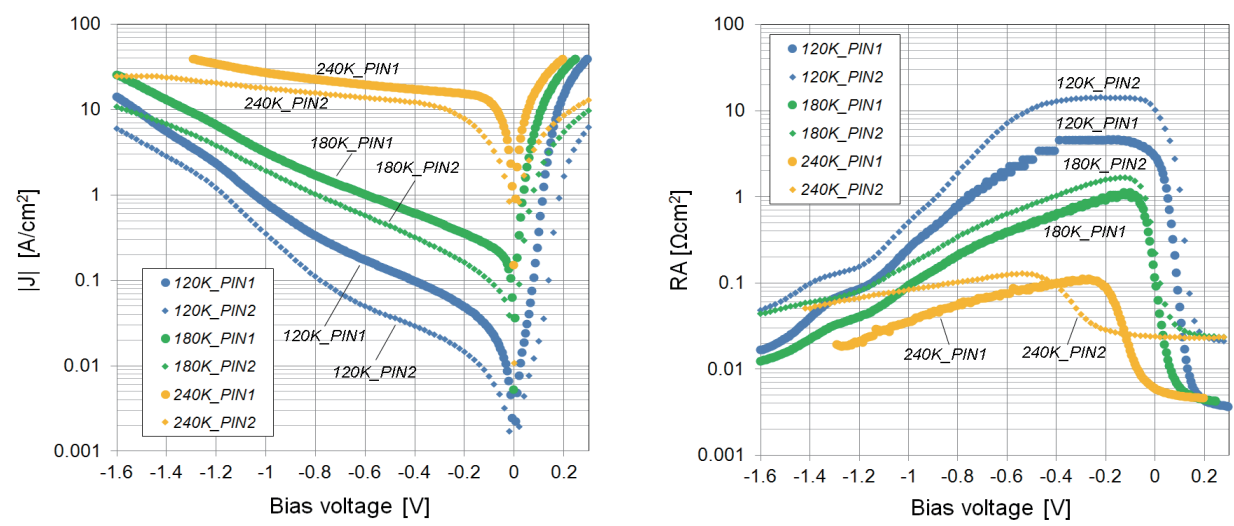

Fig. 2 (left). (Color online) Dark current density-voltage characteristics of type-II SL photodiodes at different temperatures.

Fig. 3 (right). (Color online) Differential resistance-area products versus bias voltage of type-II SL photodiodes at different temperatures.

Next, the Fourier-transformed IR (FTIR) photoluminescence (PL) spectra were measured to obtain the variation of the effective bandgap $\left(E_{\mathrm{g}}\right)$ of our structure with temperature and to confirm the existence of the inter-bandgap states, which we have taken into account in the dark current modelling. To measure FTIR PL spectra, we used a Bruker spectrometer Vertex $80 \mathrm{v}$ in a step-scan mode together with an external chamber for experiments with an additional modulated beam (the scheme of the setup and more details can be found in ref. 13). This configuration allows us to make all the measurements with the complete optical path totally under vacuum $\left(1.5 \times 10^{2} \mathrm{~Pa}\right)$, which is an important advantage in the case of making measurements in the mid- and far-IR regions where a number of atmospheric gas absorption bands can disturb the detected signal intensity. In these measurements, a liquid-nitrogen-cooled MCT photodetector was used. The pump beam was provided by the $660 \mathrm{~nm}$ line of a semiconductor laser diode and then mechanically chopped at a frequency of $275 \mathrm{~Hz}$. A phase-sensitive detection of the optical response was performed using a lock-in amplifier. 
In Fig. 4, the FTIR PL spectrum obtained (at $20 \mathrm{~K}$ ) for the PIN InAs(10 ML)/ $\mathrm{GaSb}(10 \mathrm{ML})$ sample without any electrical contacts is shown. In this graph, three features can be easily recognized. First, the peak at the energy higher than $700 \mathrm{meV}$ should be connected to recombine in the forbidden gap of the doped GaSb layer. Second, that observed at $\sim 225 \mathrm{meV}$ has been recognized as a fundamental PL between the first electron and the first heavy hole levels in InAs/GaSb SLs. The weakest signal measured for the energies lower than $100 \mathrm{meV}$ was associated with carrier recombination thru the defect states located in the energy gap of the investigated SL. Qualitatively similar results have been observed before in the electroluminescence (EL) spectra for the long-wavelength IR (LWIR) devices. ${ }^{(14)}$ The inset of this graph shows the temperature dependence of the fundamental transition in the SL. Owing to the doped GaSb cap layers, the PL signal was obtained only for low temperatures (up to $80 \mathrm{~K}$ ). Nevertheless, these values seem to be in good agreement with the energies presented by Klein et al. (which were obtained from $\lambda_{50 \% \text { cutoff }}$ of the absorption spectrum data). ${ }^{(12)}$

Thus, the variation of the effective bandgap $\left(E_{\mathrm{g}}\right)$ with temperature $(T)$, which was used in our calculation, is the same as that given by Klein et al.

$$
E_{\mathrm{g}}=0.234-3.1 \cdot 10^{-4} \cdot \frac{T^{2}}{T+270}[\mathrm{eV}]
$$

The intergap state emission and the interesting small blue shift of the main peak with temperature will be the subjects of a different contribution.

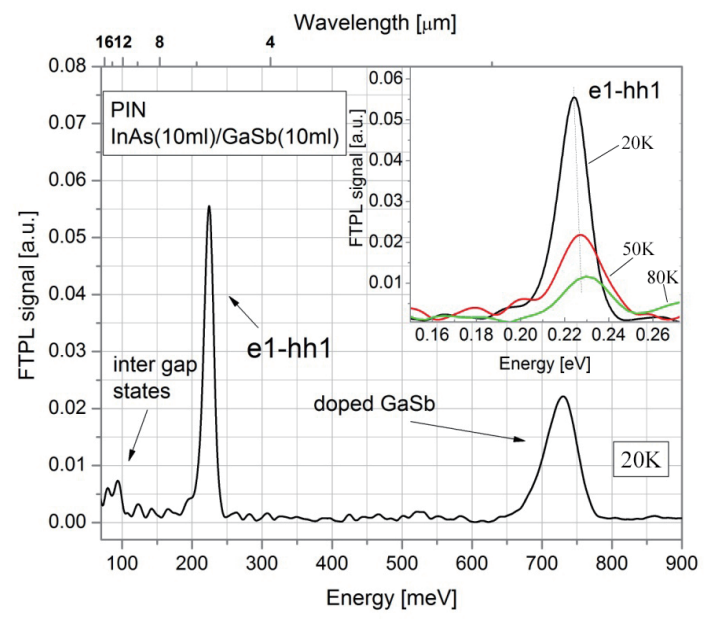

Fig. 4. (Color online) FTIR PL spectra of InAs $\operatorname{IngML}_{10} / \mathrm{GaSb}_{10 \mathrm{ML}}$ SL sample. 


\section{Modelling of Current-Voltage Characteristics}

To explain the current-voltage characteristics of the MWIR type-II SLS pin photodiodes, a bulk material model with an effective bandgap of the SL material is used. We assumed here that only two minibands essentially affect the electronic properties of the diode, namely, $\mathrm{C} 1$ and HH1. Additionally, we have taken into account the band anisotropy and adapted it to the commonly used Shockley relations for the single pn junction $^{(7-11)}$ (the second junction is isotype and is usually neglected, because of its negligibly small resistance in this case).

In general, our model is based on the standard assumption that the photodiode dark current density can be found as a superposition of several mechanisms (see Fig. 5):

$$
J_{\text {dark }}=J_{\text {diff }}+J_{\mathrm{gr}}+J_{\mathrm{btb}}+J_{\text {tat }}+J_{R \text { shunt }},
$$

i.e., diffusion $\left(J_{\text {diff }}\right)$, generation-recombination $\left(J_{\mathrm{gr}}\right)$, band-to-band tunnelling $\left(J_{\mathrm{btb}}\right)$, and trap-assisted tunnelling $\left(J_{\text {tat }}\right)$. The remaining mechanisms are current owing to the shunt resistance ( $J_{R \text { shunt }}$ originates from the surface and bulk leakage current) and $R_{\text {series }}$. The first fundamental component, $J_{\text {diff, }}$ where we assumed the reflective contact configuration, ${ }^{(15,16)}$ is given by

$$
J_{\text {diff }}=q n_{i}^{2}\left(\frac{D_{\mathrm{e}}}{N_{\mathrm{a}} L_{\mathrm{e}}} \tanh \frac{x_{\mathrm{p}}}{L_{\mathrm{e}}}+\frac{D_{\mathrm{h}}}{N_{\mathrm{d}} L_{\mathrm{h}}} \tanh \frac{x_{\mathrm{n}}}{L_{\mathrm{h}}}\right) \times\left[\exp \left(\frac{q V}{k T}-1\right)\right],
$$

where $q$ is the electron charge, $k$ is Boltzmann's constant, $T$ is the absolute temperature, $L_{\mathrm{e}, \mathrm{h}}$ is the electron/hole diffusion length, $D_{\mathrm{e}, \mathrm{h}}$ is the diffusivity $\left(L_{\mathrm{e}, \mathrm{h}}\right.$ and $D_{\mathrm{e}, \mathrm{h}}$ are connected with the diffusion lifetime $\tau_{\mathrm{e}, \mathrm{h}}$ and mobilities $\mu_{\mathrm{e}, \mathrm{h}}$ by Einstein relations), $N_{\mathrm{a}, \mathrm{d}}$ denotes acceptor/donor doping concentrations, and $x_{\mathrm{n}, \mathrm{p}}$ are the thicknesses of the $\mathrm{n}, \mathrm{p}$-type regions. The intrinsic carrier concentration $n_{i}$ can be approximated by

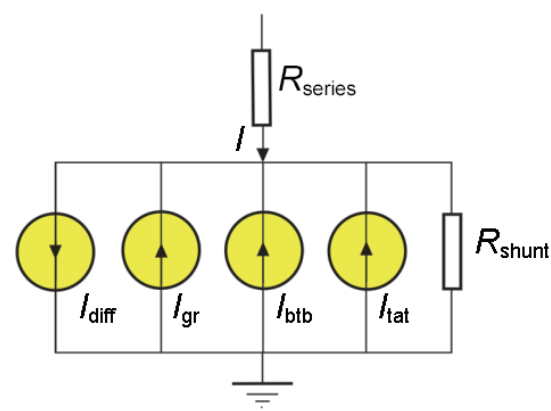

Fig. 5. (Color online) Equivalent circuit diagram. 


$$
n_{i}^{2}=N_{\mathrm{c}} N_{\mathrm{v}} \exp \left(-\frac{E_{\mathrm{g}}}{k T}\right)
$$

where the density of states of electron/holes $\left(N_{\mathrm{c}, \mathrm{v}}\right)$ is defined as

$$
N_{\mathrm{c}, \mathrm{v}}=2\left(\frac{k T m_{\mathrm{e}, \mathrm{h} \text { DOS }}^{*}}{2 \pi \hbar}\right)^{3 / 2}
$$

and the density of states of effective masses $\left(m_{\mathrm{e}, \mathrm{h} \text { DOS }}^{*}\right)$ is defined as

$$
m_{\mathrm{e}, \mathrm{h} D O S}^{*}=\left(m_{\mathrm{c} 1, \mathrm{hh} 1 / /}^{* 2} m_{\mathrm{c} 1, \mathrm{hh} 1 \perp}^{*}\right)^{1 / 3}
$$

whereas the reduced mass $\left(m_{\mathrm{red}}^{*}\right)$ in the $\mathrm{C} 1-\mathrm{HH} 1$ transition is taken as

$$
m_{\mathrm{red}}^{*-1}=m_{\mathrm{c} 1 / /}^{*-1}+m_{\mathrm{hh} 1 / /}^{*-1}
$$

because of the nonnegligible oscillator strength only in the in-plane direction. ${ }^{(17)}$

This causes the C1-HH1 band-to-band tunneling to take the form of

$$
J_{\mathrm{btb}}=\frac{\sqrt{2 m_{\mathrm{red}}^{*} q^{3} E V}}{4 \pi^{2} E \hbar \sqrt{E_{\mathrm{g}}}} \exp \left(-\frac{4 \sqrt{2 m_{\mathrm{red}}^{*}} E_{\mathrm{g}}^{3 / 2}}{3 q E \hbar}\right),
$$

where, for an abrupt pn junction, the electric field $E$ can be approximated as ${ }^{(4)}$

$$
E=\left[\frac{2 q}{\varepsilon_{0} \varepsilon_{\mathrm{s}}}\left(\frac{E_{\mathrm{g}}}{q}-V\right) \frac{N_{\mathrm{a}} N_{\mathrm{d}}}{N_{\mathrm{a}}+N_{\mathrm{d}}}\right]^{1 / 2} .
$$

Under a strong built-in field, the chance that an electron-hole pair becomes separated and collected at the electrode is much higher than that for a pair outside the depletion layer. The generation-recombination current, $J_{\mathrm{gr}}$, is a major current source in diodes with a high density of defects in the depletion region. We can approximate it as

$$
J_{\mathrm{gr}}=\frac{q n_{i} w}{\tau_{\mathrm{gr}}} \frac{2 \sinh \left(\frac{q V}{2 k T}\right)}{\frac{q\left(V_{\mathrm{b}}-V\right)}{k T}} f(b),
$$

where $f(b)$ is a complicated expression involving a trap level and an applied voltage, ${ }^{(9)}$ and $w$ is the depletion width that depends on the applied bias $V$ and the built-in potential $V_{\mathrm{b}} \mathrm{as}^{(4)}$ 


$$
w=\sqrt{\frac{2 \varepsilon_{0} \varepsilon_{\mathrm{r}}}{q} \frac{N_{\mathrm{d}}+N_{\mathrm{a}}}{N_{\mathrm{a}} N_{\mathrm{d}}}\left(V_{\mathrm{b}}-V\right)} .
$$

The second mechanism bounded with states inside the bandgap is the trap-assisted tunnelling. Similarly to our previous paper, ${ }^{(18)}$ we assumed that it is responsible for the tunnelling-like behaviour in the bias voltage from about -0.4 to $-0.6 \mathrm{~V}$. The simplified formalism, introduced by Kinch ${ }^{(19)}$ and Yang et al., ${ }^{(20)}$ has shown that

$$
J_{\text {tat }}=\frac{q^{2} m_{\mathrm{t}}^{*} M^{2} N_{\mathrm{t}} V}{8 \pi \hbar^{3}\left(E_{\mathrm{g}}-E_{\mathrm{t}}\right)} \exp \left[-\frac{4 \sqrt{2 m_{\mathrm{t}}^{*}}\left(E_{\mathrm{g}}-E_{\mathrm{t}}\right)^{3 / 2}}{3 q E \hbar}\right],
$$

where $N_{\mathrm{t}}$ is the activated trap density, $m_{\mathrm{t}}^{*}$ is the effective tunnelling mass (generally different from $m_{\text {red }}^{*}$ ), $E_{\mathrm{t}}$ is the effective trap energy level, measured from the valence band edge, and $M^{2}$ is the matrix element associated with the trap potential, assumed to be $10^{23}$ $\mathrm{eV}^{2} \mathrm{~cm}^{3}$.

Additionally, we took into consideration the shunt resistance, which is clearly seen in the low-temperature regime (see Fig. 3). In this case, we assumed bias voltage independence. A similar assumption was made for the $R_{\text {series}}$, but taking it into account is more difficult because of the connection of this mechanism to all the rest (see Fig. 5). To avoid difficulties in solving a nonlinear problem at every $I-V$ point, one can use the following simple technique:

1. calculate all components of dark current without series resistance ( $V$ in the relations above means applied voltage on the ideal structure, i.e., without series resistance)

2. calculate the voltage drop on a series resistance using the current value estimated in the previous point, from the simple Ohm's law $\left(R_{\text {series }}\right.$ can be estimated from the forward bias voltage region of a differential resistance-voltage characteristic for each temperature), and

3. add voltages used in the 1 st and calculated in the 2 nd point. This gives us the voltage drop that is connected to the previously calculated $J_{\text {dark }}$, which can be compared now with the experimental $I-V$ point.

As one can see, the voltage drop on the entire structure is calculated, not assumed to be given as an input parameter. We should underline here that this technique is correct only for the situation, where no exclusion effect is observed -i.e., where increasing $|V|$ always causes increasing $\left|J_{\text {dark }}\right|$. It is also worthy mentioning here that assuming $R_{\text {series }}=0$ might cause serious over- or underestimation of some fitting parameters under the HOT regime. ${ }^{(21)}$ One of the clearly visible effects connected to this problem is related to the deformation of $R_{\text {shunt }}$. This plot should be symmetric if $R_{\text {series }}$ would be much lower than $R_{\text {device. }}$. Because it is not true for all bias voltage points, all components of $J_{\text {dark }}$ are tensioned (effectively shifted to higher values of $|V|$ ), especially in the forward bias part of the $I-V \mathrm{~s}$. The values of the parameters used in a device modelling are the same as those in ref. 18. 


\section{Results and Discussion}

The model briefly described in our previous paper ${ }^{(18)}$ and more detailed in $\S 4$ assumes that there are eight not well-known parameters: $m_{\text {red }}^{*}, m_{\mathrm{t}}^{*}, E_{\mathrm{t}}, N_{\mathrm{t}}, \tau_{\mathrm{diff}}, \tau_{\mathrm{gr}}, R_{\text {shunt }}$, and $R_{\text {series. }}$. Each of them can be obtained through a fitting to the experimental data. For example, $R_{\text {series }}$ should limit the dark current in the linear part of a forward bias voltage [see Figs. 6(b) and 7(b), at $V=0.3 \mathrm{~V}$ ] in a device with a single junction dominance. Similarly, we predict that the tunneling-like behaviour in a high reverse bias voltage [see Figs. 6(b) and 7(b) for a region from about -0.9 to $-1.6 \mathrm{~V}$ ] should be related to the transition between the closest minibands with the highest population of levels (in our

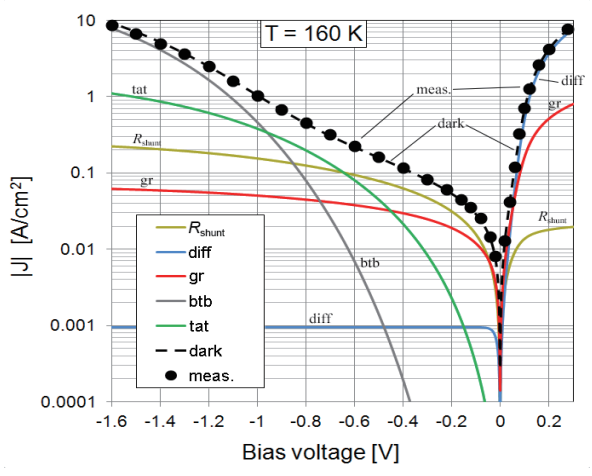

(a)

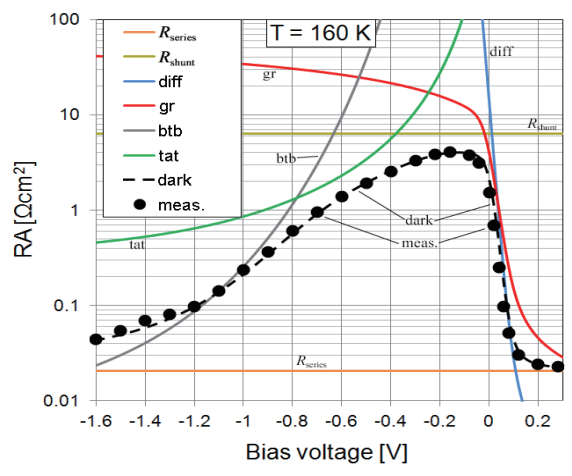

(b)

Fig. 6. (Color online) Example of measured and modelled characteristics of the PIN_2 MWIR $\mathrm{InAs}_{10 \mathrm{ML}} / \mathrm{GaSb}_{10 \mathrm{ML}}$ type-II SL photodiode at $160 \mathrm{~K}$ : dark current density (a) and resistance-area product (b) vs bias voltage.

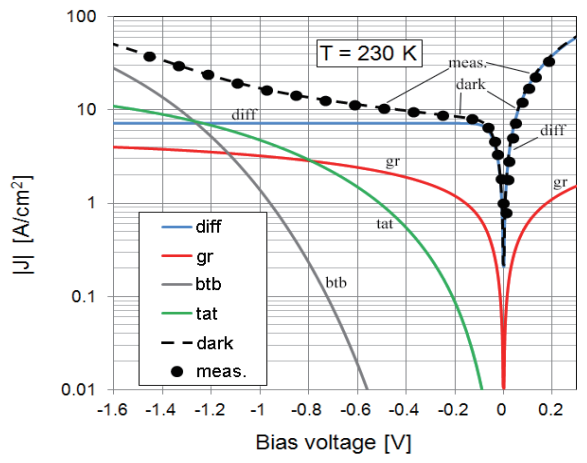

(a)

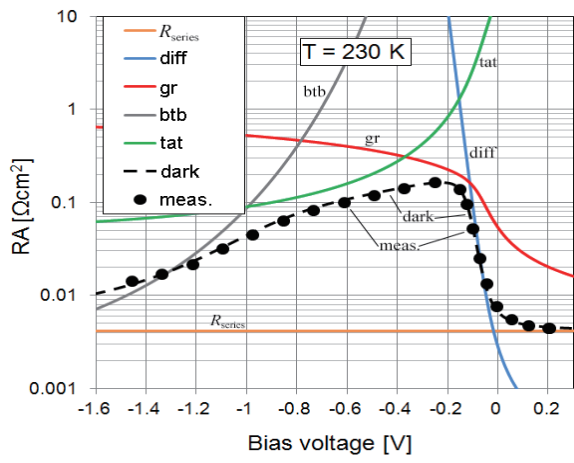

(b)

Fig. 7. (Color online) Example of measured and modelled characteristics of the PIN_1 MWIR $\mathrm{InAs}_{10 \mathrm{ML}} / \mathrm{GaSb}_{10 \mathrm{ML}}$ type-II SL photodiode at $230 \mathrm{~K}$ : dark current density (a) and resistance-area product (b) vs bias voltage. 
case, $\mathrm{C} 1$ and HH1). Because the single, not well-known parameter in eq. (8) is $m_{\text {red }}^{*}$ and the band-to-band mechanism dominates in this bias region, we can treat the fitting result of this parameter as a "measured" value. However, this point of view should be taken as a rough estimation, at least, of the two assumptions. First of all, we assumed that $R_{\text {series }}$ is bias voltage independent, which might not be true. Second, we assumed that we know the potential barrier profile. Despite this model's weaknesses, a comparison with a much more difficult calculation and more direct measurements gives unexpectedly convergent results. Thus, our fittings for a few MWIR InAs ${ }_{10 \mathrm{ML}} / \mathrm{GaSb}_{10 \mathrm{ML}}$ samples (with essentially different $R_{\text {shunt }}$ and $R_{\text {series }}$ values) gave an average $m_{\text {red }} \approx 0.0148 m_{0}$, nearly independent of temperature (it is the subject of a different contribution).

The electron effective masses of the MWIR InAs $\mathrm{x}_{\mathrm{X} \text { ML }} / \mathrm{GaSb}_{\mathrm{x} \_\mathrm{ML}}$ T2SLs (or very similar) for the in-plane direction $\left(m^{*}{ }_{\mathrm{c} 1 / /}\right)$ were measured by several authors. Suchalkin et al. ${ }^{(22)}$ reported $m_{\mathrm{c} 1 / /}^{*}$ equal from about $0.025 m_{0}$ to $0.028 m_{0}$ depending on the sample, Omaggio et al. ${ }^{(23)}$ estimated it from about $0.027 m_{0}$ to $0.033 m_{0}$, and Fuchs et al., (24) based on magneto-optics measurements and $8 \times 8 \mathrm{Kp}$ calculation, obtained $0.0235 \mathrm{~m}_{0}$. Additionally, they estimated $m_{\text {hhl// }}^{*}$ as about $0.0342 m_{0}$. Putting average values into eq. (7), one can see that $m_{\text {red }}^{*}$ should be on the order of $0.015 m_{0}$, which agrees very well with our results.

\section{Conclusions}

In this work, we analyzed the dark current of MWIR type-II InAs/GaSb SL pin photodiodes in the temperature range from 120 to $240 \mathrm{~K}$ and bias voltage from -1.6 to $+0.3 \mathrm{~V}$. Our calculations were mainly focused on obtaining the reduced mass value from current-voltage characteristics for the $\mathrm{C} 1-\mathrm{HH} 1$ transition in $\mathrm{InAs}_{10 \mathrm{ML}} / \mathrm{GaSb}_{10 \mathrm{ML}}$ samples. We showed that simplified formalism, based on a bulk material model, is sufficient for estimating such reduced mass, but an essential step in this procedure is connected with taking into account the anisotropy of the band structure and the series resistance. Our fittings gave $m_{\text {red }} \approx 0.0148 m_{0}$, which agree well with much more complex simulations and cyclotron resonance measurements.

\section{Acknowledgements}

This study was carried out with financial support from the National Science Center (grant DEC-2012/05/N/ST7/01057) and the Polish Ministry of Sciences and Higher Education Key Project POIG.01.03.01-14-016/08, "New Photonic Materials and their Advanced Applications".

\section{References}

1 G. A. Saihalasz, R. Tsu and L. Esaki: Appl. Phys. Lett. 30 (1977) 651.

2 L. L. Chang, N. Kawai, G. A. Saihalasz, R. Ludeke and L. Esaki: Appl. Phys. Lett. 35 (1979) 939.

3 A. Rogalski and P. Martyniuk: Infrared Phys. Technol. 48 (2006) 39. 
4 A. Rogalski: Infrared Detectors (CRC Press, Boca Raton, 2009) 2nd ed., p. 808.

5 P. Martyniuk and A. Rogalski: Opto-Electron Rev. 21 (2013) 239.

6 F. Szmulowicz, H. J. Haugan, S. Elhamri and G. J. Brown: Phys. Rev. B 84 (2011) 14.

7 C. L. Canedy, E. H. Aifer, J. H. Warner, I. Vurgaftman, E. M. Jackson, J. G. Tischler, S. P. Powell, K. Olver, J. R. Meyer and W. E. Tennant: Infrared Phys. Technol. 52 (2009) 326.

8 A. Hood, D. Hoffman, B. M. Nguyen, P. Y. Delaunay, E. Michel and M. Razeghi: Appl. Phys. Lett. 89 (2006) 233513.

9 J. Nguyen, D. Z. Ting, C. J. Hill, A. Soibel, S. A. Keo and S. D. Gunapala: Infrared Phys. Technol. 52 (2009) 317.

10 D. R. Rhiger, R. E. Kvaas, S. F. Harris and C. J. Hill: Infrared Phys. Technol. 52 (2009) 304.

11 C. Cervera, K. Jaworowicz, H. Ait-Kaci, R. Chaghi, J. B. Rodriguez, I. Ribet-Mohamed and P. Christol: Infrared Phys. Technol. 54 (2011) 258.

12 B. Klein, E. Plis, M. N. Kutty, N. Gautam, A. Albrecht, S. Myers and S. Krishna: J. Phys. D. Appl. Phys. 44 (2011) 5.

13 M. Motyka, G. Sek, J. Misiewicz, A. Bauer, M. Dallner, S. Hofling and A. Forchel: Appl. Phys. Express 2 (2009) 126505.

14 Q. Yang, C. Pfahler, J. Schmitz, W. Pletschen and F. Fuchs: Proc. SPIE 4999 (2003) 448.

15 H. J. Hovel, R. K. Willardson and A. C. Beer: Semicond. Semimet. 11 (1975) 254.

16 B.-M. Nguyen: Ph.D. Thesis, Northwestern University, Evanston, Illinois, USA (2010).

17 T. V. C. Rao, S. V. Nair, H. E. Ruda, J. Antoszewski, J. B. Rodriguez, E. Plis, S. Krishna and L. Faraone: Semicond. Sci. Technol. 27 (2012) 7.

18 J. Wrobel, P. Martyniuk, E. Plis, P. Madejczyk, W. Gawron, S. Krishna and A. Rogalski: Proc. SPIE 8353 (2012) 835316.

19 M. A. Kinch: Semicond. Semimet. 18 (1981) 313.

20 Q. K. Yang, F. Fuchs, J. Schmitz and W. Pletschen: Appl. Phys. Lett. 81 (2002) 4757.

21 J. Wróbel, P. Martyniuk and A. Rogalski: Adv. Opt. Technol. 2012 (2012) 5.

22 S. Suchalkin, G. Belenky, S. P. Svensson, B. Laikhtman, D. Smirnov, L. C. Tung and S. Bandara: J Appl. Phys. 110 (2011) 043720.

23 J. P. Omaggio, R. J. Wagner, J. R. Meyer, C. A. Hoffman, M. J. Yang, D. H. Chow and R. H. Miles: Semicond. Sci. Technol. 8 (1993) S112.

24 F. Fuchs, E. Ahlswede, U. Weimar, W. Pletschen, J. Schmitz, M. Hartung, B. Jager and F. Szmulowicz: Appl. Phys. Lett. 73 (1998) 3760. 